\title{
GROUPS WITH A FINITE NUMBER OF SYLOW $\Lambda$-SUBGROUPS
}

\author{
EUGENE SCHENKMAN
}

\begin{abstract}
Two theorems on groups with a finite number of Sylow subgroups are proved.
\end{abstract}

THEOREM 1. If for some set of primes $\Lambda$ the group $G$ has a finite number of Sylow $\Lambda$-subgroups, then $G$ is a finite extension of a $\Lambda^{\prime}$-extension of $O_{\Lambda}(G)$.

THEOREM 2. Let the set of all primes be a disjoint union of sets of primes $\Lambda$ such that for each $\Lambda$, the group $G$ has a finite number of Sylow $\Lambda$-subgroups. Then the set $T$ of periodic elements of $G$ is a normal subgroup and $T / R$ is an $F C$ group with $R$ denoting $\Pi_{\Lambda} O_{\Lambda}(G)$.

Various authors (cf. [1, pp. 362-369])-Baer, Neumann, Haimo, Dickman, Eremin-have studied FC groups and conditions of finiteness for certain classes of elements or subgroups. Černikov studied thin FL groups (cf. [2, p. 139]). This note considers groups with finiteness conditions on Sylow subgroups and aims to prove the two theorems mentioned above.

Proof of Theorem 1. For $i=1, \ldots, n$, let $P_{i}$ be the Sylow $\Lambda$-subgroups of $G$ and let $N_{i}=\Re\left(P_{i}\right)$ be their normalizers. If $D=\cap_{i=1}^{n} N_{i}$, then the index $|G: D|$ is finite since $\left|G: N_{i}\right|=n$ is finite. Since $N_{i}$ has exactly one Sylow $\Lambda$-subgroup, the same is true of $D$. In fact the Sylow $\Lambda$-subgroup of $D$ is $O_{\Lambda}(G)$ and Theorem 1 follows.

Proof of TheOREM 2. As in the proof and with the notation of Theorem 1, $O_{\Lambda}(G)$ is of finite index in $P_{i}$; for in $N_{i}, P_{i} / O_{\Lambda}(G)$ is isomorphic to $P_{i} D / D$ and $|G: D|$ is finite. Thus $G / O_{\Lambda}(G)$ has a finite number of finite Sylow $\Lambda$-subgroups for each $\Lambda$. It follows from the theorem of Dickman that all the Sylow $\Lambda$-subgroups of $G / O_{\Lambda}(G)$ are contained in a finite normal subgroup and hence that the Sylow $\Lambda$-subgroups of $G$ generate a finite extension of $O_{\Lambda}(G)$. Thus $T$ is a normal subgroup and $T / R$ is an FC group as is asserted in Theorem 2.

After submitting this paper the author learned that Professor Derek Robinson was aware of these results for the cases where $\Lambda$ consists of one prime.

\section{BIBLIOGRAPHY}

1. G. Baumslag, Reviews on infinite groups. Parts 1, 2, Amer. Math. Soc., Providence, R. I., 1974. MR $50 \# 2311$.

2. D. J. S. Robinson, Finiteness conditions and generalized solvable groups. Part 1, Ergebnisse der Mathematik und ihrer Grenzgebiete, Band 62, Springer-Verlag, Berlin and New York, 1972. MR 48 \#11314.

Department of Mathematics, Purdue University, West Lafayette, Indiana 47907

Received by the editors September 25, 1975.

AMS (MOS) subject classifications (1970). Primary 20E20; Secondary 20F15.

Key words and phrases. Sylow subgroup, FC group. 